\title{
Is there a need for non-drug treatments in headaches?
}

Cephalalgia

2016, Vol. 36(12) II0I-1102

(C) International Headache Society 2016

Reprints and permissions:

sagepub.co.uk/journalsPermissions.nav DOI: $10.1177 / 0333102416641639$

cep.sagepub.com

(S)AGE

\section{Jean Schoenen}

Dear reader,

Despite undisputed therapeutic breakthroughs in recent decades, migraine still ranks amongst the top ten human diseases with the greatest number of years lived with disability (1). Several factors explain this top ranking. First, as migraine does not decrease longevity, it can cause disability and needs to be managed during the greater part of the subject's lifespan. Second, migraine is widely undertreated (2), and even the most disabled subjects may not consult with or not be informed by their practitioner of the optimal management options. Third, there is no cure for migraine, and the available preventative (i.e. disease-modifying) drug treatments are efficient in no more than $30-50 \%$ of patients (3), and one of the most effective of such treatments is abandoned by one patient out of every four because of its disabling side effects (4). The therapeutic score is higher for specific acute anti-migraine drugs, although this largely depends on outcome measure (headache relief versus freedom) and route of administration (oral versus parenteral) (3).

In the pathophysiological cascade leading to the migraine attack, activation and sensitisation of the trigeminovascular nociceptive system can be symbolised as being located at the narrow exit of a funnel and involves a restricted number of pathogenic actors, among which calcitonin gene-related peptide (CGRP), the target of the recently introduced immune therapies for migraine. By contrast, the factors that are at the broad entrance of the pathogenic funnel and induce activation of the trigeminovascular system are multiple and combine a number of genetic liability loci, epigenetic factors and hormonal influences, abnormal information processing and mitochondrial energy reserve, cortical and subcortical mechanisms. Moreover, all of these can vary between patients, over a patient's life and over the migraine cycle (5), illustrating the fact that disease-modifying therapy for migraine remains a formidable challenge for which one single intervention will never be optimal.

Except for invasive neurostimulation in refractory chronic cluster headache patients, non-drug treatments have shown no superiority over the available pharmaceuticals to date. However, they can have some advantages over such pharmaceuticals. They are well tolerated overall and can be combined with conventional drug therapies, and some of them are effective both for preventative and acute treatment. Unfortunately, at present, a major handicap of several non-drug therapies is that they lack evidence-based proof of efficacy. This has been critically addressed by the contributors to this special issue, whom I would like to thank for the quality of their reviews and for their patience.

This special issue will start with a review of the possible pathophysiological targets of non-pharmacological therapies of migraine. Nutraceuticals and diets will then be examined, followed by cognitive behavioural therapies ('psychoceuticals'). The review on physical therapies in headaches ('physicoceuticals') will precede four articles on neurostimulation methods ('electroceuticals'). The first three of these articles will address invasive neurostimulation methods that are to be restricted to refractory headache patients: hypothalamic deep brain and sphenopalatine ganglion stimulation in cluster headache and invasive pericranial nerve interventions. The fourth of these articles summarises the present evidence regarding non-invasive neurostimulation methods in migraine. Last but not least, a review on integrated multidisciplinary care for difficult patients will close out this issue.

We hope that you will find here useful information on the various interventions that are alternatives to drug therapies and, for some of them, can be combined

Headache Research Unit, University of Liège, Citadelle Hospital, Liège, Belgium

\section{Corresponding author:}

Jean Schoenen, Headache Research Unit, University of Liège, Citadelle Hospital, Liège, B-4000, Belgium.

Email: jschoenen@ulg.ac.be 
with drugs. As mentioned, the multifactorial nature of migraine pathogenesis and clinical experience with recommended drug treatments suggest that combining different therapeutic strategies is likely to provide superior benefit to our patients, although admittedly this remains largely to be confirmed in randomised controlled trials.

\section{Jean Schoenen}

February 2016

\section{Declaration of conflicting interests}

The authors declared no potential conflicts of interest with respect to the research, authorship, and/or publication of this article.

\section{Funding}

The authors received no financial support for the research, authorship, and/or publication of this article.

\section{References}

1. Global Burden of Disease Study 2013 Collaborators. Global, regional, and national incidence, prevalence, and years lived with disability for 301 acute and chronic diseases and injuries in 188 countries, 1990-2013: a systematic analysis for the Global Burden of Disease Study 2013. Lancet 2015; 386: 743-800.

2. Miller S and Matharu MS. Migraine is underdiagnosed and undertreated. Practitioner 2014; 258(1774): 19-24.

3. Tfelt-Hansen P and Olesen J. Taking the negative view of current migraine treatments: the unmet needs. CNS Drugs 2012; 26: 375-382.

4. Bussone G, Diener H-CC, Pfeil J, et al. Topiramate 100 $\mathrm{mg} /$ day in migraine prevention: a pooled analysis of double-blind randomised controlled trials. Int $J$ Clin Pract 2005; 59: 961-968.

5. Sándor PS, Agosti R and Schoenen J. Migraine. Pain Clin Updates 2001; 9: 1-4. 